\title{
THE JUDGMENT OF SPORT JURISDICTION BODIES ON DOPING CASES
}

\author{
Konstantinos Konstantinidis ${ }^{1}$ \\ School of Physical Education and Sport Science, National and Kapodistrian \\ University of Athens Greece
}

Dimitrios Panagiotopoulos ${ }^{2}$

National and Kapodistrian University of Athens, Greece

panagiod@otenet.gr

\section{Abstract}

This paper studies how sport jurisdiction bodies encounter doping cases when they are called upon, hear the parties involved, and decide in the first or second instance on the sanctions imposed. In any case, the attitude of the sports authorities plays a crucial role not only in the implementation of the regulations, the issuance of decisions and therefore the creation of Lex Sportiva, but also in the attitude of the sports community towards doping. The present study is based on the use of interpretive and jurisprudential review as the core of the methodological approach. Then, there is a comparison between the decisions of the National and International Federations, the WADA (World Anti-doping Agency) and the decisions of the CAS. (Arbitration Court for Sports). The research showed how the attitude of the bodies differs not only in the severity of the crises but also in the imposed sanctions. Through CAS decisions, it is clear that as the authority of the bodies increases, so does the rigor they display. In particular, WADA always appears stricter in doping cases and often brings them before CAS demanding stricter sanctions than those already in place. On the other hand, the International Federations often appear less strict, while the national federations often show

${ }^{1}$ Candidate Doctor of Sports Law, School of Physical Education and Sport Science, National and Kapodistrian University of Athens Greece.

Ethnikis Antistasis 41, Dafni 17237, Greece.

${ }^{2}$ Professor of Law, National and Kapodistrian University of Athens, Greece, Attorney-at-Law.

4, Veranzerou Str., 10677, Athens, Greece. 
the most lenient attitude. Furthermore, there is a difference in the decisions and argumentation of CAS, which comes either from the different legal culture of the referees or from the more tolerant interpretation concerning the athlete objective responsibility. In short, it is observed that the treatment of doping cases lacks stability.

\section{Keywords}

Sports; sports law; doping; sport bodies; sport jurisdiction; Court of Arbitration for Sport; WADA

\section{CONTENTS}

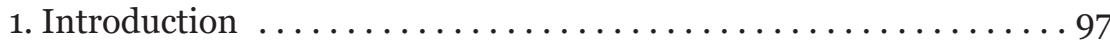

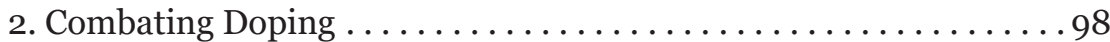

3. International bodies vs national bodies . . . . . . . . . . . 99

3.1. The case of Irene Kokkinariou . . . . . . . . . . . . . . . . 99

4. International body assertion . . . . . . . . . . . . . 100

4.1. The case of Tatyana Chernova ................ 100

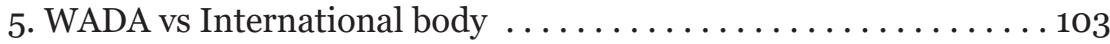

5.1. The case of José Paolo Guerrero . . . . . . . . . . . 103

5.2. The case of Damar Robinson ... . . . . . . . . . . . 104

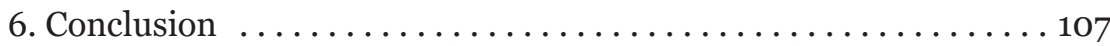

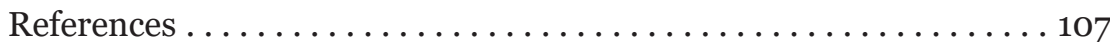

\section{Introduction}

There is no doubt that victory is the sole purpose of top level sport. On this basis, many athletes, in order to improve their performance and achieve victories, resort to doping. ${ }^{3}$ It is well-known that doping not only threatens the athletes' health and well-being, but also corrupts fair competition, promotes fictitious patterns, and enhances the degeneration of the athletic ideal. ${ }^{4}$ In the field of international and national sports law, sport and political bodies are in a constant effort

${ }^{3}$ N. Cox, Victory with Honour or Victory at All Costs: Towards Principled Justification for anti-Doping Rules in Sport, 22 Dublin ULJ 19 (2000).

4 Dimitrios P. Panagiotopoulos, Zografenia Kallimani, Implementation of WADA Code in the Greek Sports Legal Order, IV(1-2) e-Lex Sportiva Journal 135-139 (2016). 
to combat and limit this ever-growing phenomenon. As a whole, doping cases are addressed to the competent sport courts, which are called upon to impose sanctions, to hear the parties involved, or/and to adjudicate in second instance on appeals. ${ }^{5}$ Several cases override athletic jurisdiction and are brought before civil courts as well. ${ }^{6}$ In all cases, the approach of the sport governing and judicial bodies plays a crucial role in the implementation of lex sportiva and the ruling process, but also in the attitude of the sports community towards doping and the methods of combating it. Thus, the purpose of this research is not to comment on the verdict of the CAS (Court of Arbitration for Sport), but, to highlight and interpret the different approach in the judgment of sport bodies, by presenting and comparing their positions and arguments in doping cases.

\section{Combating Doping}

WADA (World Anti-Doping Agency) defines doping, as a criminal act of possession, manipulation, use or attempted use of substances and methods as defined and prohibited by WADA, as well as behaviors that obstruct or violate the sampling and control procedures prescribed by the Code, and any possible complicity or association with persons accused of doping. ${ }^{7}$ The WADA Code and the UNESCO International Convention against Doping, together with the regulations of the International Olympic Committee, are the cornerstones of the entire sports community in combating doping. These provisions define both the regulations of the international and national federations, and the relevant national legislative acts. It is worth noting that over the years there has been a more harmonized relationship between the Code and the regulations of the federations. However, there are often divergences between federations and international sports organizations. These divergences are identified at two levels: (a) the severity of the judgment,

5 T. Kavanagh, The Doping Cases and the Need for the International Court of Arbitration for Sport (CAS). 22 UNSWLJ 721 (1999).

${ }^{6}$ D. Panagiotopoulos, International Sports Rules' Implementation-Decisions' Executability: The Bliamou Case, 15 Marq. Sports L. Rev. 1 (2004).

7 WADA World Anti-Doping Code, art. 2, at 17-24, 2015. 
and then consequently (b) the imposition of the sanctions envisaged. Both categories of divergences appear mainly when a doping case arrives at the judicial bodies, and in particular in front of the CAS, where the intentions of the sport bodies can be observed through their testimonies. In this view, there has been a comparison of the decisions and positions of National and International Federations and the WADA through the jurisdictional committees and before the CAS. The comparative criteria for interpreting the judgment of each institution are the argumentative approach and the proposed sentences. Below are presented some examples which support these arguments.

\section{International bodies vs national bodies}

\subsection{The case of Irene Kokkinariou ${ }^{8}$}

Very often there is a lack of cohesion between the national and international jurisdictional organs. The case of the Greek swimmer Irene Kokkinariou reveals this incoherency. The athlete participated in the official athlete's biological passport program (ABP), and was found guilty of doping offenses, there was a difference between the SEGAS and IAAF judgment.

\section{The facts}

Specifically, after examining four 2006-2009 samples taken in the context of hematological parameters and nine samples in the period 2009-2011 under the ABP program, abnormal blood values were found in the athlete's samples; the IAAF post-mission expert report called on her for explainations. The athlete's explanations were insufficient and the IAAF informed her through SEGAS that she would be sentenced to a four-year sentence under IAAF Rule 40.6, which applies to aggravating circumstances that may increase the penalty period. SEGAS briefed the sportswoman on the IAAF decision, citing part of the regulation stating that if convicted, the sentence would be reduced to two years. The athlete denied the allegations and requested a hearing from the SEGA Disciplinary Committee.

${ }^{8}$ See CAS 2012/A/2773, http://jurisprudence.tas-cas.org/Shared Documents/ 2773.pdf. 


\section{National body assertion}

Following the hearing, SEGAS issued a decision alleging that the athlete violated Rule $32.2^{9}$ on the use or attempted use of a prohibited substance or prohibited method, and imposed the two-year penalty provided for by Rule $40.2,{ }^{10}$ without recognizing a violation of 40.6 as this regulation is not applied in this case.

\section{International body assertion}

The IAAF then appealed to the CAS, where neither the SEGAS nor the athlete participated. In this case, the IAAF's attitude towards that of SEGA was stricter, as the International Federation considered that Regulation 40.6 was the one to be applied, in contrast to the Greek Federation, though it had twelve controls in its hands. In 2006-2011 it stated that this case is not a case of repeated use but: “... a case involving an abnormal variation in the blood profile according to those provided by the IAAF Blood Testing Protocol...”, essentially stated that because the samples were taken for $\mathrm{ABP}$, they could not be subject to a violation of Rule 40.6.

\section{CAS verdict}

The court, after examining the parties' positions and background to the case, rejected the argument of the National Federation and ruled in favor of the IAAF, imposing four-year exclusion on the athlete.

\subsection{The case of Tatyana Chernova ${ }^{11}$}

\section{The facts}

Similarly, in the case of heptathlon athlete Tatyana Chernova, the athlete was found guilty of violating Rule 32.2(b) of the IAAF Rules: "Use or Attempted Use by an Athlete of a Prohibited Substance or a Prohibited Method." Again, these allegations were based mainly on

9 IAAF Competition Rules 2011, Rule 32.

${ }^{10}$ IAAF Competition Rules 2011, Rule 40.

${ }^{11}$ See CAS 2016/O/4469, http://jurisprudence.tas-cas.org/Shared Documents/ 4469.pdf. 
the participation of the athlete in the ABP program for the period 2009-2014 with the collection of 19 blood samples. Though the first of these 19 samples, was collected on the occasion of the 2009 IAAF International Championship that took place in Berlin, and when it was retested in 2013, the athlete was found positive for the anabolic steroid called "oral turinabol".

\section{National anti-doping body assertion}

As a result, but with a delay of almost two years on 20 January 2015, the Russian Disciplinary Anti-Doping Committee (RUSADA), imposed a 2 years ineligibility period from 22 July 2013 to 21 July 2015 and disqualified all her results from 15 August 2009 (date of the sample collection) to 14 August 2011.

\section{International body assertion}

A few months later IAAF filed an appeal (still pending) against this decision of RUSADA (CAS 2015/A/4050), requesting an increased sentence both for the ineligibility of the athlete and also for her results in more events. At the same time, a group of experts analyzed the ABP profile of the athlete on an anonymous basis and found that the hematological profile is highly likely to be a result of the use of prohibited substances. Hence, the IAAF Anti-Doping Administrator informed the All-Russia Athletics Federation (ARAF) that they will put charges against the athlete unless she could prove otherwise. The athlete failed to provide convincing explanations, and at the same time upon request of the IAAF, the Expert Panel issued the "Joint Expert Opinion" concluding that it is highly possible that the usage of prohibited substances from the athlete took place. Further on, its pushing and strict strategy against any possible doping violation, IAAF suspended ARAF's membership and took over the case calling the athlete to stand in front of a CAS sole arbitrator as a first instance. Moreover, according to Rule 42 IAAF set ARAF as a respondent to its claim since, according to IAAF, ARAF turned to be incapable of resolving the case on time and thus they are responsible for part of the arbitration fees. At this point, it can be seen that IAAF leaves no space to anyone for 
deflecting away from its Rules the Code and the battle against doping. Additionally, IAAF asked before the CAS Court Office that the athlete should provide relevant information regarding her possible doping usage before August 14 2009, the date of the first sample, pushing her more. However, the Sole Arbitrator did not allow this since it was profoundly possible that such an addition would further harm the athlete. During the hearing, the IAAF raised the issue of whether the athlete had had a second anti-doping rule violation, and suggested that neither the principle of proportionality nor the principle of fairness should be applied in this case. By the same token, the IAAF was seeking for the strictest award of 4 years of ineligibility and disqualification of all competitive results from 14 August 2009 until 5 February 2016.

\section{CAS verdict}

The Sole Arbitrator after examining all evidence, allegations and facts, partially upheld the IAAF's claim by imposing an ineligibility period of three years and eight months starting from 5 February 2016 and disqualify any result from 15 August 2011 to 22 July 2013. Again, on this case the international federation left no margin for any attempt to treat any possible doping violation lenient. They did not even hesitate to disengage their member federation (ARAF) because of its loose handling and accelerate the process.

Likewise, it is well known that WADA has a clear view of doping and considers how an athlete is responsible for entering its body, in other words the athlete bears objective responsibility, and therefore he has been subject to doping and must be subject to strict penalties, ${ }^{12}$ WADA's rigorous stance is also confirmed in a recent doping case by José Paolo Guerrero.

${ }_{12}$ A. Duval et al. The world anti-doping code 2015: asser international sports law blog symposium, 16(1-2) The International Sports Law Journal 99-117 (2016). 


\section{WADA vs International body}

\subsection{The case of José Paolo Guerrero ${ }^{13}$}

\section{The facts}

The soccer player was found to be positive in the presence of the cocaine metabolite benzoelectin ("BZE") after an in-competition AntiDoping control match against the Argentinian National Team for the Qualifier Rounds of the 2018 FIFA World Cup Russia.

\section{International federation assertion}

The FIFA Disciplinary Committee imposed a 30-day exclusionary penalty under the interim measures. The player then lodged an appeal with the FIFA Appeals Board, seeking the decision of the Disciplinary Committee to be quashed. The Appeals Board dismissed the player's appeal and upheld the interim measures I had been imposed on him. At the start of the proceedings against the FIFA jurisdictions, the football player filed his position in writing with the evidence in his possession. Following the hearing, the FIFA Disciplinary Committee examined written submissions and supporting evidence and imposed a one-year suspended sentence on the player.

The player then turned to the appeals committee, which reduced his sentence to six months. However, the player, following the procedure and not accepting the verdict of the FIFA institutions, addressed the CAS, by completing an appeal.

\section{WADA assertion}

Immediately after the player's recourse to the CAS, WADA requested to intervene in the process as permitted by the CAS code as well managed: (a) the position of the player seeking the annulment of the FIFA disciplinary decision and the annulment of the WADA appeal; (b) the position of FIFA seeking the cancellation of the player's appeal and imposition of a six-month ban on (c) the position of WADA seeking to dismiss the player's appeal, annul FIFA's decision and impose a maximum penalty of two years as set forth in the FIFA Regulation.

${ }_{13}$ See CAS 2018/A/5546 \& CAS 2018/A/5571, http://jurisprudence.tas-cas.org/ Shared Documents/5546, 5571.pdf 


\section{CAS verdict}

Finally, after reviewing all the facts, the CAS concluded that the player's appeal should be rejected and that FIFA and WADA's positions were partially accepted, imposing a total penalty of 14 months.

\subsection{The case of Damar Robinson ${ }^{14}$}

\section{The facts}

In another case, the case of Jamaican track and field athlete Damar Robinson, there was involvement of both the national bodies and WADA. At this case after Mr. Robinson's participation in the National Junior Championship of Jamaica, he was selected for doping control.

\section{National body assertion}

His urine sample proved to be positive and the same happened with his sample B. Because of this, JADCO (Jamaican Anti-Doping Commission) informed him that he was provisionally suspended from all official competitions immediately. A few weeks later Mr. Robinson accepted a scholarship from Cloud County Community College in Concordia, Kansas, U.S.A., and in January 2014, started competing at the National Junior College Athletic Association and National Collegiate Athletic Association events, representing his college. At this point, it is important to mention that none of these associations is signatory to WADA and therefore obliged to operate under the WADA code. On February 2014, the Disciplinary Panel of JADCO decided to suspend him for one year according to Article 10.5.2 of the Jamaican Anti-Doping Rules $^{15}$ for acting with No Significant Fault or Negligence. Mr. Robinson appealed but the Appeals Panel rejected his grounds and upheld the first decision. Furthermore, the Appeals Panel found that Mr. Robinson had failed to prove that he had provided "Substantial Assistance" under Article 10.5.3. However, JADCO even if they could appeal asking for a stricter decision, they did not do so.

${ }_{14}$ See CAS 2014/A/3820, http://jurisprudence.tas-cas.org/Shared\%2oDocu ments/3820.pdf.

${ }^{15}$ See JADCO Anti-Doping Rules, Article 10.5.2. 


\section{WADA assertion}

On November 2014, WADA filled an appeal to CAS with respondents both Mr. Robinson and JADCO. WADA stated that according to the code, the sanction for Mr. Robinson's offense is two years and not one as JADCO decided, since he is not meeting any of the attenuating conditions of Article 10.5. Besides, he "failed to establish any link between any specific product (whether given to him by his Coach or otherwise) and the prohibited substance in his system." Moreover, WADA profoundly mentioned that regardless Mr. Robinson's relationship with his coach this was "incompatible with his personal duty under the World AntiDoping Code and the JADCO [Anti-Doping] Rules". ${ }^{16}$ Finally, WADA argued that Mr. Robinson's participation to the National Junior College Athletic Association and National Collegiate Athletic Association events was not allowed according to his suspension.

\section{CAS Verdict}

Finally, the court decided to set aside the first decision, suspend the athlete for two years and disqualify all the results retrieved from events organized by bodies that are bound by WADA Code only.

\section{Considerations}

In the above cases, it can be seen that the judgment of the judicial authorities changes according to their jurisdiction. As jurisdiction grows, so does the severity of the judgment on doping cases. In some of the above cases, there were examples where the national body would show a more lenient attitude while the international body demonstrates a stricter one and finally WADA would show the strictest. At the same time international bodies and WADA more often, would also stand before CAS by filling appeals not only regarding the athletes but also the subsistent bodies who were incapable to apply the anti-doping rules at a level that would protect the integrity of sport according to the purpose and scope of WADA Code. ${ }^{17}$ For this, the rules clearly give the bodies the right to take control over cases where the national body did not act

\footnotetext{
16 See CAS 2014/A/3820, Ibid, 7.

${ }_{17}$ See World Anti-Doping Code, 11(2018).
} 
within time limits or with the necessary caution like the case of Tatyana Chernova mentioned before. Moreover, as it was shown in the cases of José Paolo Guerrero and Damar Robinson, WADA can anytime bring a case to CAS if they do not agree with the decision rendered at the previous stages, investigating most of the times additional facts that could draw extra measures for the parties. This is an extra evidence to support WADA's "surveillance culture" in view to more restrained sport bodies. ${ }^{18}$ The above study raises several questions as to: a) whether different opinions and positions of the institutions regarding the same cases can contribute to a fair and equal treatment of all parties involved, b) whether the rights of athletes are safeguarded and c) whether a sense of justice is provided. This is because one would expect that based on the harmonization of the regulations of the federations and national legal systems with the anti-doping rules of WADA, there would be a common and coherent approach. As it has been presented in the past concerning the Bliamou case $\mathrm{e}^{19}$ and has shown in some of our case studies, the sport bodies using their autonomy, and their political influence power, regarding their jurisdiction, can proportionally exert pressure on both the subsistent bodies and before the CAS, requiring stricter or more lenient treatment in the cases of its interest. One could state that WADA, which has proved over the time to stand against any doping case with a more authoritarian and rigid manner, is an exception. Moreover, sports authorities have full jurisdiction over the sports affairs, even the disciplinary ones as we are discussing now, while the arbitration clause prohibits those involved in sports from having to resort to civil courts even in cases of unlawful decisions. ${ }^{20}$

${ }_{18}$ J.K. Park, Governing doped bodies: the world anti-doping agency and the global culture of surveillance. 5(2) Cultural Studies, Critical Methodologies 174-188 (2005).

19 Panagiotopoulos, D. International Sports Rules' Implementation [...]. Op. cit., p. 11-12. (2004).

${ }^{20}$ Dimitrios P. Panagiotopoulos, Arbital Jurisdiction in Sports Activities. IV(1-2) e-Lex Sportiva Journal 20-34 (2016). 


\section{Conclusion}

Within the context of sport bodies jurisdiction in doping case several issues emerge: a) the need for careful application of the principle of justice in order to develop an institutional framework that is not only credible, but also homogeneous; b) the political influence on the sport governing bodies once again confirms the unbalanced and heterogeneous nature of the contractual relationships among sport stakeholders; c) there is a deep subjectivity that characterizes sports organizations, even on the same issue; d) the role of the institutions is crucial and, therefore, it is imperative that they respond to doping cases on the basis of a common framework that will be respected in the light of an independent judiciary, with a view to protecting sports, respecting the rights of athletes and recognizing their peculiar organization, as appropriate and effective.

\section{REFERENCES}

1. Cox N., Victory with Honour or Victory at All Costs: Towards Principled Justification for anti-Doping Rules in Sport, 22 Dublin ULJ 19 (2000).

2. Dimitrios P. Panagiotopoulos, Arbital Jurisdiction in Sports Activities, IV(1-2) e-Lex Sportiva Journal 20-34 (2016).

3. Dimitrios P. Panagiotopoulos, Zografenia Kallimani, Implementation of WADA Code in the Greek Sports Legal Order, IV(1-2) e-Lex Sportiva Journal 135-139 (2016).

4. Duval A. et al., The world anti-doping code 2015: asser international sports law blog symposium, 16(1-2) The International Sports Law Journal 99-117 (2016)

5. Park J.K., Governing doped bodies: the world anti-doping agency and the global culture of surveillance. 5(2) Cultural Studies, Critical Methodologies 174-188 (2005).

6. Kavanagh T., The Doping Cases and the Need for the International Court of Arbitration for Sport (CAS), 22 UNSWLJ 721 (1999).

7. Panagiotopoulos D., International Sports Rules' ImplementationDecisions' Executability: The Bliamou Case, 15 Marq. Sports L. Rev. 1 (2004). 